\title{
Why some people discount more than others: baseline activation in the dorsal PFC mediates the link between COMT genotype and impatient choice
}

\author{
Lorena R. R. Gianotti ${ }^{1}{ }^{*}{ }^{\dagger}$, Bernd Figner ${ }^{2,3 t}$, Richard P. Ebstein ${ }^{4}$ and Daria Knoch ${ }^{\mathbf{*}}$ \\ 1 Social and Affective Neuroscience, Department of Psychology, University of Basel, Basel, Switzerland \\ ${ }^{2}$ Center for Decision Sciences, Columbia University, New York, NY, USA \\ ${ }^{3}$ Department of Psychology, University of Amsterdam, Amsterdam, Netherlands \\ ${ }^{4}$ Department of Psychology, National University of Singapore, Singapore
}

\section{Edited by:}

Daeyeol Lee, Yale University School of Medicine, USA

\section{Reviewed by:}

NaYoung So, University of

Washington, USA

R. McKell Carter, Duke University, USA

\section{*Correspondence}

Lorena R. R. Gianotti and Daria Knoch, Social and Affective Neuroscience,

Department of Psychology, University of Basel, Birmannsgasse 8, CH-4050

Basel, Switzerland.

e-mail: Iorena.gianotti@unibas.ch;

daria.knoch@unibas.ch

${ }^{\dagger}$ Lorena R. R. Gianotti and Bernd Figner have contributed equally to this work.
Individuals differ widely in how steeply they discount future rewards. The sources of these stable individual differences in delay discounting (DD) are largely unknown. One candidate is the COMTVal158Met polymorphism, known to modulate prefrontal dopamine levels and affect DD. To identify possible neural mechanisms by which this polymorphism may contribute to stable individual DD differences, we measured 73 participants' neural baseline activation using resting electroencephalogram (EEG). Such neural baseline activation measures are highly heritable and stable over time, thus an ideal endophenotype candidate to explain how genes may influence behavior via individual differences in neural function. After EEG-recording, participants made a series of incentive-compatible intertemporal choices to determine the steepness of their DD. We found that COMT significantly affected DD and that this effect was mediated by baseline activation level in the left dorsal prefrontal cortex (DPFC): (i) COMT had a significant effect on DD such that the number of Val alleles was positively correlated with steeper DD (higher numbers of Val alleles means greater COMT activity and thus lower dopamine levels). (ii) A whole-brain search identified a cluster in left DPFC where baseline activation was correlated with DD; lower activation was associated with steeper DD. (iii) COMT had a significant effect on the baseline activation level in this left DPFC cluster such that a higher number of Val alleles was associated with lower baseline activation. (iv) The effect of COMT on DD was explained by the mediating effect of neural baseline activation in the left DPFC cluster. Our study thus establishes baseline activation level in left DPFC as salient neural signature in the form of an endophenotype that mediates the link between COMT and DD.

Keywords: intertemporal choice, delay discounting, endophenotype, resting EEG, individual differences, COMT genotype

\section{INTRODUCTION}

Leading a healthy and successful life requires individuals to forego sooner-smaller rewards (SSs), immediate gratification, to reach longer-term goals in the form of later-larger rewards (LLs). It is typical and often rational that future (i.e., delayed) rewards are somewhat discounted (Green and Myerson, 2004). However, excessively steep delay discounting (DD) - i.e., the tendency to strongly discount future rewards as a function of their delay and thus choose tempting SSs over even substantially larger LLs - has been implicated in suboptimal life outcomes in the financial, academic, and health domain (Mischel et al., 2011) and in different psychiatric disorders including substance (ab)use (Bickel et al., 2007; MacKillop et al., 2011), pathological gambling (Reynolds, 2006), and ADHD (Scheres et al., 2010).

Not only clinical populations, but also healthy individuals differ widely in how steep or shallow their DD is, i.e., how strongly they de-value rewards as a function of the length of the delay. Individuals' steepness of DD has been shown to be stable and to have trait-like characteristics (Beck and Triplett, 2009; Casey et al., 2011; Mischel et al., 2011; Odum, 2011). Moreover, steepness of DD in monetary intertemporal decisions predicts individual differences in impulsive everyday-life behaviors, including smoking, relationship infidelity, drug use, and overeating (Reimers et al., 2009). Surprisingly, however, the sources of these stable individual differences are still largely unknown (Shamosh et al., 2008; Olson et al., 2009; Peters and Buchel, 2011). Given the pervasiveness of intertemporal choices in important life domains and the role of steep DD in problematic behaviors, it is important not only to identify the sources of individual differences but also to understand the mechanisms by which they influence people's decisions (Peters and Buchel, 2010). Here, we apply a novel neuro-genetic individual differences approach to identify such a candidate mechanism in DD.

A recent twin study found a substantial genetic component for DD, explaining up to $50 \%$ of the individual differences in DD (Anokhin et al., 2011). While this finding implies a sizable genetic 
influence, the study was not designed to identify which specific genes contribute to determining an individual's steepness of DD. A molecular genetics study by Boettiger et al. (2007) found that the Val158Met polymorphism of the catechol- $O$-methyltransferase (COMT) gene is associated with $\mathrm{DD}$, as individuals with a greater number of Val alleles exhibited steeper DD (but, see Paloyelis et al., 2010). The COMT gene is relevant to DD as it encodes a major enzyme that degrades dopamine, thus influencing dopamine levels in a dose-dependent relationship, particularly in the prefrontal cortex (Tunbridge et al., 2004). The Val allele (compared to the Met allele) is associated with higher enzymatic activity and thus lower prefrontal cortex dopamine levels (Chen et al., 2004).

Efforts to directly relate specific genes to specific behaviors have often yielded mixed results, sometimes failing to be replicated (Meyer-Lindenberg and Weinberger, 2006). Importantly, even when specific genes for certain behavioral phenotypes could be discovered, the question about the mechanisms - i.e., how these genes influence behavior - still remained unanswered. We can find an answer to this question by taking so-called brain-based endophenotypes into account. The idea behind this approach is that genes influence an individual's behavior through their effects on the brain (Meyer-Lindenberg and Weinberger, 2006). This provides a mechanistic understanding of the contribution of specific gene variants to individual differences in neurobiology and behavior that goes beyond simple biomarker identification (Braver et al., 2011).

A promising candidate for the brain-based endophenotype approach is neural baseline activation measured with electroencephalography while the participant is at rest and not engaged in any specific task (resting EEG), because neural baseline oscillatory activity is both under genetic influence and stable over time. In fact, resting EEG measures are among the most heritable traits in humans (De Geus, 2010), with heritability estimates of 70-96\% (Smit et al., 2005; Zietsch et al., 2007; van 't Ent et al., 2009). Various studies have investigated the intraindividual stability of resting EEG, revealing test-retest reliabilities of up to 0.8 over a period of up to 5 years (Dunki et al., 2000; Smit et al., 2005; Napflin et al., 2007). Moreover, intraindividual specificity (i.e., the extent to which an EEG pattern uniquely belongs to a given person, compared to others) has been shown to be highly reliable with a recognition rate of up to 99\% (Dunki et al., 2000; Napflin et al., 2007). This allows EEG to capture stable dispositional individual differences in neural functioning at rest, akin to a "neural fingerprint."

The goal of this study was to use neural baseline activation as an endophenotype in order to identify key neural traits through which the COMT Val158Met genotype contributes to individual differences in steepness of DD. For the identification of the neural traits, a whole-brain voxel-by-voxel approach was applied (thus not restricting the analyses to any a priori hypothesized region). Figure 1 shows a model of the tentative mediation mechanism under investigation. An observable effect of COMT genotype on DD (COMT $\rightarrow$ DD; path c) is hypothesized to be due to the following two legs of an indirect path: COMT genotype influences neural baseline activation (COMT $\rightarrow$ Baseline activation; path a) and, in turn, neural baseline activation influences steepness of $\mathrm{DD}$ (Baseline activation $\rightarrow \mathrm{DD}$; path $\mathrm{b}$ ). The crucial test for our mediation model is thus to show that paths $\mathrm{a}$ and $\mathrm{b}$ mediate the direct effect of COMT genotype on DD steepness.

\section{MATERIALS AND METHODS PARTICIPANTS}

Seventy-three healthy Swiss men (mean age $=25.7$ years, $\mathrm{SD}=5.0$ years) participated in the study. All were right-handed and reported no past or present neurological or psychiatric disorders, nor alcohol or drug abuse. COMT genotype distribution was in Hardy-Weinberg equilibrium $\left(\chi^{2}=1.25, P=0.26\right.$; see Methods in Appendix for details). Participants were remunerated with a flat fee of 40 Swiss francs (CHF; CHF $1 \approx$ USD 0.90) in addition to a variable payment depending on participants' choices. The study was approved by the local Ethics Committee.

\section{EXPERIMENTAL DESIGN}

After obtaining written informed consent, participants completed the state and trait forms of the positive and negative affect schedule (PANAS; Watson et al., 1988) and were asked about the number of

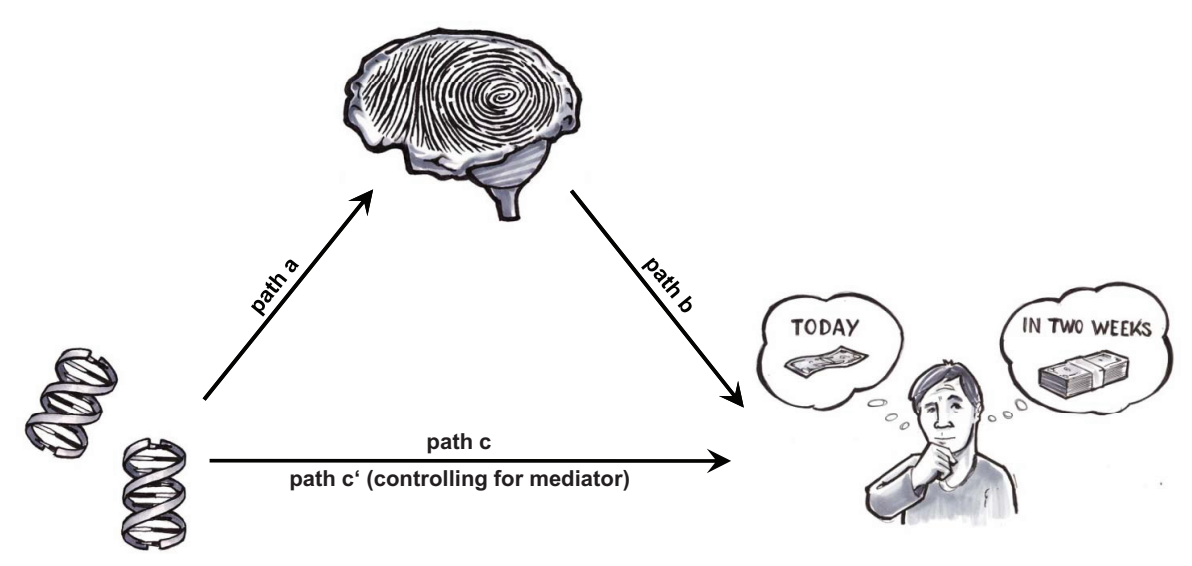

FIGURE 1 | Brain-based endophenotype approach as applied in the present study: is the relationship between specific genes and intertemporal choice mediated by neural baseline activation, a stable neural trait akin to a "neural fingerprint?" If so, such a "neural fingerprint" provides a mechanism of how genes can influence steepness of DD. 
years of education. Participants were seated in a sound- and electrically shielded EEG-recording chamber with dim illumination and intercom connection to the experimenter. They were instructed that EEG-recording was to be done during resting with open and closed eyes. The protocol consisted of 20-s eyes open followed by 40 -s eyes closed, repeated five times (such a protocol guarantees minimal fluctuations in participants' vigilance state). Only data from the 200-s eyes closed condition were analyzed. Participants were then asked to perform a mouthwash to collect buccal epithelial cells for the preparation of DNA. Thirty minutes after resting EEG-recording, all participants received written instructions for the intertemporal choice task (including comprehension questions to ensure full understanding). After the intertemporal choice task, participants filled out the Barratt Impulsivity Scale-II (BIS-II; Patton et al., 1995).

\section{ELECTROENCEPHALOGRAPHIC RECORDING AND RAW DATA PROCESSING}

A 128-channel EEG was recorded using the Geodesic Sensor Net system (Electrical Geodesic, USA). The sampling rate was $250 \mathrm{~Hz}$ (16 bit precision; bandwidth: $0.1-100 \mathrm{~Hz}$ ), and the vertex electrode $(\mathrm{Cz})$ served as recording reference. Amplifier gains and zeros were measured prior to each recording session. Impedances were kept below $30 \mathrm{kOhm}$. Independent component analysis (ICA) was applied, and ICA components that clearly accounted for vertical and horizontal eye movements were removed from the EEG without topographic distortion. In addition to the rejection of sweeps where any channel exceeded the amplitude of $\pm 100 \mu \mathrm{V}$, the data were visually inspected to reject remaining artifacts, using a moving, non-overlapping 2-s window. The electrodes on the outermost circumference (chin and neck) were excluded and 111 electrodes were maintained for subsequent analysis. For each participant, channels exhibiting substantial noise were interpolated using a $3 \mathrm{D}$ spherical spline interpolation procedure. The EEG data were then recomputed against the average reference. On average, there were $68 \pm 29$ 2-s epochs available per participant. Fast Fourier Transformation (using a square window) was applied to each epoch and channel to compute the spectra with $0.5 \mathrm{~Hz}$ resolution. For each participant, the spectra for each channel were averaged over all epochs. Power spectra were integrated for the following seven independent frequency bands (Kubicki et al., 1979): delta (1.5$6 \mathrm{~Hz})$, theta $(6.5-8 \mathrm{~Hz})$, alpha1 $(8.5-10 \mathrm{~Hz})$, alpha2 $(10.5-12 \mathrm{~Hz})$, beta1 $(12.5-18 \mathrm{~Hz})$, beta2 $(18.5-21 \mathrm{~Hz})$, and beta3 $(21.5-30 \mathrm{~Hz})$. Confirming the stability and consistency of participants' vigilance achieved with our EEG-recording protocol, Tables A2 and A3 in Appendix present Spearman rank correlation coefficients for the short term test-retest reliability of the present data.

\section{INTRACORTICAL SOURCE LOCALIZATION ANALYSIS}

Standardized low-resolution brain electromagnetic tomography (sLORETA; Pascual-Marqui, 2002) was used to calculate the intracortical electrical sources that generated the scalp-recorded activity in each of the seven frequency bands. sLORETA is a distributed source localization technique that solves the inverse problem without assuming an a priori number of underlying sources and computes electric neural activity as standardized current density (unit: amperes per square meter, $\mathrm{A} / \mathrm{m}^{2}$ ). The sLORETA solution space consisted of 6239 voxels (voxel size: $5 \mathrm{~mm} \times 5 \mathrm{~mm} \times 5 \mathrm{~mm}$ ) and was restricted to cortical gray matter, as defined by the digitized Montreal Neurological Institute (MNI) probability atlas. In order to reduce confounds that have no regional specificity, such as total power inter-subject variability, a global normalization of the whole-brain sLORETA images was carried out. Before statistical analyses, the sLORETA images were further log-transformed.

\section{INTERTEMPORAL CHOICE TASK}

Thirty minutes after resting EEG, participants made a series of 36 incentive-compatible binary intertemporal choices, each between an SS and an LL. SS amounts ranged from CHF 15 to CHF 85 (pseudo-randomly drawn from a normal distribution with a mean 45 , capped at the lower end at 15 and 85 at the upper end); LL amounts were determined according to a factorial design with LLs being $0.5-75 \%$ larger than SSs. Crossed with this factor, the difference between SS and LL time of delivery was 2 or 4 weeks; again crossed with the previous factors, SS time of delivery was either today or in 2 weeks. Choices were used to estimate each participant's hyperbolic discount rate $k$ that best explained their choice pattern (see Intertemporal Choice Task in Appendix). Thus, each participant's $k$ numerically described the steepness of their DD, indexing how patiently or impatiently they chose in the intertemporal choice task. Higher values of $k$ indicate steeper DD; $k$ values were square-root transformed for the analyses.

\section{DNA PREPARATION AND GENOTYPING}

Participants were instructed to rinse their mouths with $25 \mathrm{ml}$ of mouthwash and to spit the fluid into sterile 50 -ml polypropylene tubes. The samples were stored at $4^{\circ} \mathrm{C}$ if they could not be processed within $2 \mathrm{~h}$ of collection. DNA from these samples was extracted and amplified (Genetica, Zurich, Switzerland).

The Val158Met polymorphism of the COMT gene was genotyped using the SNaPshot Method (Applied BioSystems, Foster City, CA, USA; see Appendix for detailed description of the DNA preparation and genotyping procedure).

\section{DATA ANALYSES}

The goal of this study was to use neural baseline activation as an endophenotype in order to identify key neural traits through which the COMT genotype contributes to individual differences in DD steepness. Accordingly, as first step, we tested the effect of COMT genotype on DD, using linear regression. Note that for all regression analyses involving the COMT genotype, the number of Val alleles was used as predictor $(\mathrm{Met} / \mathrm{Met}=0, \mathrm{Val} / \mathrm{Met}=1$, $\mathrm{Val} / \mathrm{Val}=2$ ). Using the Bayesian information criterion (Raftery, 1995) we found that this additive model was the one that best fitted our data (compared to a quadratic model and two models which either pooled Met/Met and Val/Met or Val/Val and $\mathrm{Val} / \mathrm{Met}$ ). We then applied a whole-brain voxel-wise correlation approach to identify brain regions whose baseline activation correlates with $\mathrm{DD}$, separately for each EEG frequency band. To minimize Type I errors, only activation clusters of more than 10 contiguous voxels $\left(1.25 \mathrm{~cm}^{3}\right)$ exceeding corrected $P<0.05$ were considered significant. Full correction for multiple testing (for all voxels and all frequency bands) was implemented by means of a non-parametric randomization approach (Nichols and Holmes, 
2002). The non-parametric randomization approach was used to estimate empirical probability distributions and the corresponding corrected (for multiple comparisons) critical probability thresholds. Importantly, this approach captures whole-brain baseline cortical activation that predicts DD. After having identified clusters of potential neural mediators, current density was averaged across all voxels within a cluster, thus yielding one value per participant.

In the next step, a mediation analysis was conducted to test whether the association between COMT genotype and DD can be explained by the functional indirect pathway via the potential mediator. The predictor was COMT genotype, the outcome was DD, and the potential mediator was cortical baseline activation in the identified cluster. Significant mediation was defined as significance in three tests (MacKinnon et al., 2007): (i) Significant indirect path a, relating the predictor to the mediator. (ii) Significant indirect path $b$, relating the mediator to the outcome, controlling for the predictor. (iii) Significant functional mediation pathway by showing that the $\mathrm{a} \times \mathrm{b}$ cross product is significantly different from 0 ( $P<0.05$, using bootstrapping with 5000 samples; Preacher and Hayes, 2008). Bootstrapping involves the repeated extraction of samples from the data set and the estimation of the indirect effect in each resampled data set. The totality of all the estimated indirect effects permits the construction of a 95\% confidence interval for the effect size of the indirect effect. A confidence interval that does not include zero indicates a significant indirect effect and thus establishes mediation.

Following a reviewer's suggestion, we additionally confirmed our result with a related analysis approach, called mediation effect parametric mapping (MEPM; Wager et al., 2008). With this analysis, a whole-brain search was performed, testing the neural baseline activation in each voxel for mediation. As in the previous analysis, significant mediation was defined as significance in the same three tests (see above; $P<0.05$, more than 10 contiguous voxels, using bootstrapping with 5000 samples).

\section{RESULTS}

We summarize our statistical results in the path diagram in Figure 2. The bivariate relationships corresponding to the three paths (path a: COMT $\rightarrow$ Baseline activation; path b: Baseline activation $\rightarrow D D$; path $\mathrm{c}: C O M T \rightarrow D D$ ) are visualized with three separate graphs in Figures 2A-C. In the following section, we present the analyses for each of the three paths, and the test for mediation.

\section{$\mathrm{COMT} \rightarrow \mathrm{DD}$}

As depicted in Figure 2C, there is a clear dose effect of the number of Val alleles: Individuals with a higher number of Val alleles exhibited significantly steeper DD (i.e., greater impatience; path c: $C O M T \rightarrow D D$; linear regression: $D D=i_{1}+c \times C O M T+e_{1}$, where $c$ corresponds to $\beta=0.30 ; P<0.01)$. This establishes the direct effect of COMT on DD.

\section{BASELINE ACTIVATION $\rightarrow$ DD}

To investigate whether this relationship was mediated by neural baseline activation, we next, as second step, used a whole-brain voxel-by-voxel correlation approach to identify brain regions whose baseline activation correlates with DD (path b), separately for each EEG frequency band. We found that in the beta3 frequency band $(21.5-30 \mathrm{~Hz})$ a brain area of 15 voxels showed significant correlations between current density and DD $(P<0.05$, whole-brain corrected for multiple testing).

These voxels all fell into one cluster in the BA 9/10 in the left hemisphere [peak voxel: MNI $(x, y, z)-15,50,45$ in the superior frontal gyrus; Figure 2]. There is no agreement in the literature about the labeling of this area: some authors label it dorsal prefrontal cortex (DPFC, e.g., Boettiger et al., 2007), some other authors call it dorsolateral prefrontal cortex (e.g., Pizzagalli et al., 2005). Following a reviewer's suggestion, we will use the broader term "DPFC."

Previous research has shown that beta activity indicates excitatory cortical activation: beta rhythm has been shown to increase with attention (Murthy and Fetz, 1992), arousal (Bonnet and Arand, 2001), vigilance (Bouyer et al., 1987), and directly through cortical stimulation via transcranial magnetic stimulation (Paus et al., 2001). Results from simultaneously recorded PET and EEG during rest have shown a positive correlation between beta activity and cerebral blood flow (Gamma et al., 2004; Oakes et al., 2004). Moreover, Laufs et al. (2003) report predominantly positive correlations between the BOLD signal and the EEG power in the 24- to $30-\mathrm{Hz}$ bandwidth. All these results convergently suggest that beta3 activity indicates higher activation levels.

As shown in the Figure 2B, beta3 EEG activity in the identified cluster (i.e., averaged current density across voxels within the cluster) was negatively correlated with participants' DD (path b: baseline activation $\rightarrow \mathrm{DD}$; linear regression, controlling for COMT genotype: $\mathrm{DD}=\mathrm{i}_{2}+\mathrm{b} \times$ baseline activation $+\mathrm{c}^{\prime} \times \mathrm{COMT}+\mathrm{e}_{2}$, where $\mathrm{b}$ corresponds to $\beta=-0.38 ; P=0.0009)$, indicating that a lower level of baseline activation in the left DPFC was associated with steeper DD. Our findings were highly specific to the left DPFC because no significant correlations were found in any other EEG frequency bands, and in no other brain region was resting beta3 activity correlated with DD.

\section{COMT $\rightarrow$ BASELINE ACTIVATION}

As illustrated in Figure 2A, beta3 EEG activity in the identified cluster was negatively correlated with the number of Val alleles (path a: COMT $\rightarrow$ baseline activation; linear regression: baseline activation $=\mathrm{i}_{3}+\mathrm{a} \times \mathrm{COMT}+\mathrm{e}_{3}$, where a corresponds to $\beta=-0.25 ; P=0.03)$, indicating that a greater number of Val alleles was associated with a lower level of baseline activation in the left DPFC.

\section{MEDIATION TEST}

As illustrated in Figure 2, bootstrapping revealed significant mediation, meaning that baseline activation in left DPFC significantly mediated the effect of COMT genotype on DD (cross product $\mathrm{a} \times$ b: $P<0.05$; 95\% confidence interval: 0.01-0.36).

As additional evidence for mediation (in the spirit of more traditional mediation approaches comparing paths $c$ and $c^{\prime}$ ), when controlling for baseline activation in left DPFC, the path from COMT genotype to DD became non-significant (path $c^{\prime}: D D=i_{2}+b \times$ baseline activation $+c^{\prime} \times C O M T+e_{2}$, where $c^{\prime}$ corresponds to $\beta=0.21 ; P=0.07)$. 


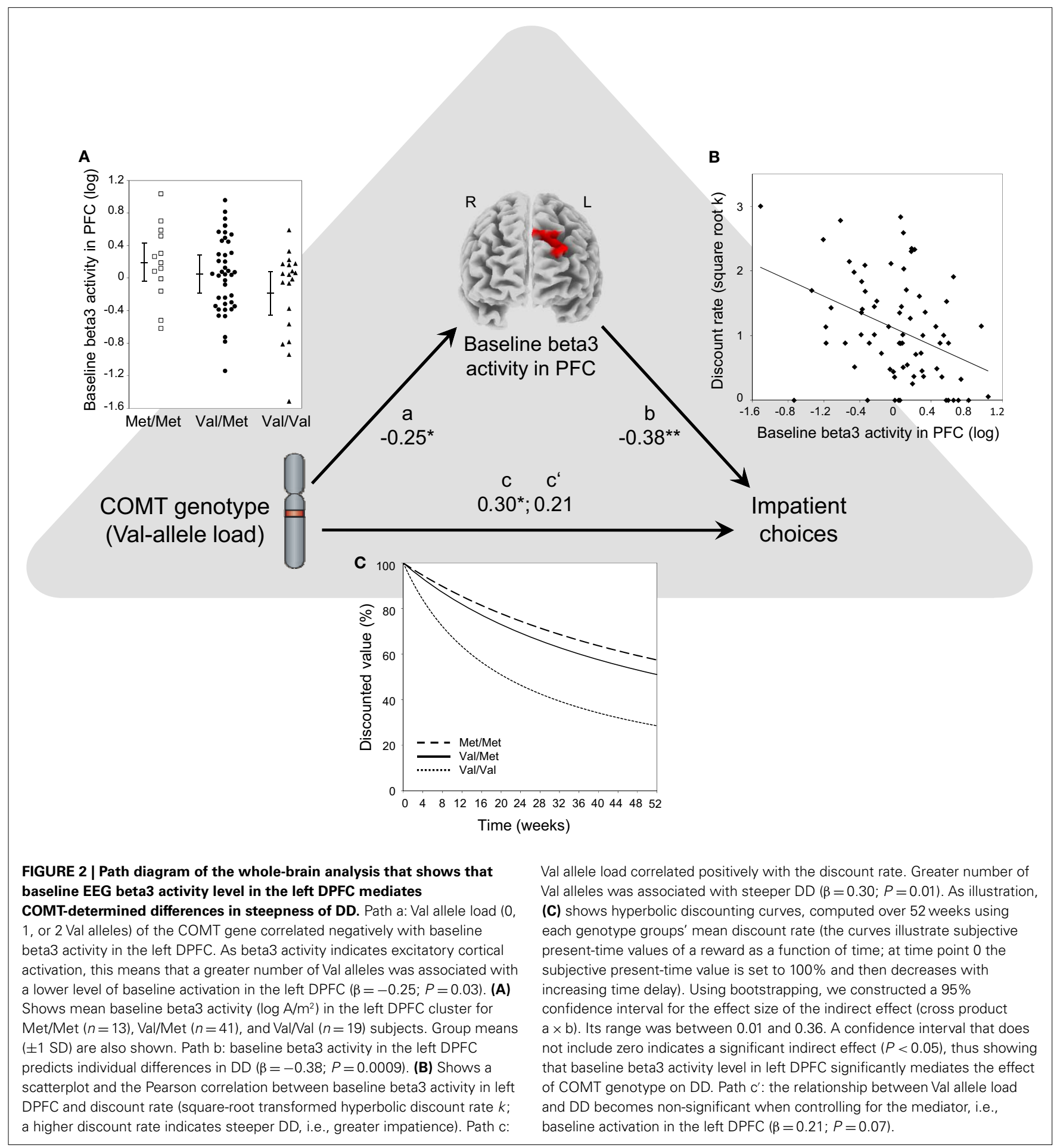

Thus, our mediation results establish baseline activation in the left DPFC as a brain-based endophenotype that acts as a mechanism by which COMT genotype influences DD steepness: individuals carrying a higher number of Val alleles show lower levels of baseline activation in the left DPFC, which in turn leads to steeper DD.

As suggested by a reviewer, we followed up with a whole-brain MEPM analysis that searches for voxels that significantly mediate

the relationship between COMT and DD. Replicating our result, we identified a region of 35 voxels in the left DPFC $(P<0.05$ for path $a$, path $b$, and the $a \times b$ mediation effect $)$. Supporting the robustness of the result, $60 \%$ of the region identified in the previous analysis overlapped with the cluster identified with the MEPM approach.

Genotype groups did not differ in terms of age, educational level, levels of pre-task affect (PANAS), and self-reported 
impulsivity (BIS-II), thus ruling out alternative explanations that the relationships between COMT genotype, DD, and baseline activation in left DPFC might have been caused by differences in these variables (for details, including Table A1 in Appendix).

\section{DISCUSSION}

People differ substantially in how steeply they discount future rewards (i.e., the steepness of their DD). These individual differences have been found not only to be stable over long time periods but to also be predictive of important life outcomes (Beck and Triplett, 2009; Kirby, 2009; Reimers et al., 2009; Casey et al., 2011; MacKillop et al., 2011; Mischel et al., 2011; Odum, 2011). While substantial individual differences are typically observed within non-clinical populations, even steeper discounting has reliably been reported for several psychiatric conditions including substance abuse, pathological gambling, and ADHD - all characterized by impulsive decision-making tendencies. Contrasting with this great ecological relevance in both non-clinical and clinical populations, relatively little is known about the sources and neural and genetic mechanisms that lead to these individual differences in DD (Shamosh et al., 2008; Olson et al., 2009; Peters and Buchel, 2010, 2011). The current study used a task-independent measure of neural activity, resting EEG. This is an important feature, because this measure provides a trait-like neural signature of an individual's neural function and is thus well suited to explain stable individual differences in behavior and bridging the gap between genotype and behavior. Across the whole-brain, our analyses identified baseline activation specifically in the left DPFC as the neuroanatomical substrate mediating the effect of COMT genotype on DD. Individuals with a higher number of Val alleles show lower levels of baseline activation in the left DPFC. Lower levels of baseline activation in the left DPFC in turn lead to steeper DD.

While DD cannot simply be equated with self-control, selfcontrol in tasks like ours is likely to contribute to more shallow DD and in this perspective shallow DD has reasonably been interpreted as an expression of greater self-control (and, vice versa, steep DD is commonly interpreted as expression of impulsivity; Monterosso and Ainslie, 1999; Shamosh et al., 2008; Casey et al., 2011). Importantly, several studies found brain regions in the DPFC - similar to the region identified in our study (albeit some of them more lateral than ours) - implicated in functions that can be interpreted as self-control processes: two recent studies found that individuals with lower levels of baseline activation in the lateral DPFC exhibited risky and social decision-making behaviors indicative of lower self-control capacity, compared to individuals with higher levels of baseline activation (Gianotti et al., 2009; Knoch et al., 2010). This area has also been implicated in self-control in fMRI studies in value-based decision-making, both in intertemporal choice and in other decision-making domains (McClure et al., 2004; Hare et al., 2009; for excellent reviews, see Carter et al., 2010; Peters and Buchel, 2011). Moreover, experimentally modulating activity in this area leads to changes in intertemporal choice behavior: decreasing the activity in the left lateral DPFC by means of transcranial magnetic stimulation leads to steeper DD (Figner et al., 2010). Combining the findings of these studies with our current results leads us to speculate that the baseline level of activation in the DPFC might reflect a "degree of self-control capacity." The higher this baseline level is, the greater the self-control capacity. Our results then suggest that one factor affecting this capacity would be the COMT genotype. As we have shown, the number of Val alleles has a significant effect on left DPFC baseline activation. This neural baseline activation in turn predicts how patiently individuals choose in an intertemporal choice task, implying individual differences in self-control capacity (however, whether such a mechanism may operate via influencing valuations, e.g., as in Hare et al., 2009, or via a more direct effect on choice as in Figner et al., 2010 is an open question that will require further research).

Clearly, COMT genotype and left DPFC baseline activation are not the only factors associated with individual differences in DD. Besides other possible genetic factors, non-genetic factors such as parental socio-economic status affect the maturation and plasticity of brain circuitries that are important for self-regulatory behaviors (Hackman et al., 2010). Moreover, recent research has found white matter integrity (Olson et al., 2009) and proportional volume of the DPFC (Bjork et al., 2009) to be related to steepness of DD. Interestingly, COMT genotype has been shown to affect prefrontal white matter integrity (Liu et al., 2010). Future studies could investigate whether prefrontal white matter integrity and neural baseline activation explain unique or shared variance of individual differences in steepness of DD and self-control capacity. Other studies could investigate which other (genetic or non-genetic) factors besides COMT genotype might affect both neural baseline activation (and DD), as COMT unlikely is the only factor determining neural baseline activation.

Our results in conjunction with previous research also shed new light on how the level of cortical baseline activation may impact activity during task performance. Using fMRI, Boettiger et al. (2007) investigated the effect of COMT genotype on DD and the role of neural activity during task performance. They found, as we did, that higher Val allele load was associated with steeper DD. However, while they observed greater left DPFC activation in Val carriers compared to Met carriers during task performance, we - using a measure of task-independent neural baseline activity - observed lower levels of baseline activation in this area in Val carriers compared to Met carriers. This suggests that the increased activity in Val carriers during task performance observed by Boettiger et al. might reflect compensatory recruitment of more neural resources.

In conclusion, one important contribution of the current study is that it establishes the effect of a stable characteristic (COMT genotype) on behavior (DD) and thus identifies a source of individual differences in a trait (steepness of DD) that is a crucial component of impulsive behaviors and self-control failure implicated in important life outcomes and different psychiatric conditions (Winstanley et al., 2006; Reimers et al., 2009; Mischel et al., 2011). In addition, the current results give novel insights into the neural mechanisms by which a specific genotype may impact a specific behavior. We identified a mediation mechanism by which a genetic trait translates into a stable neural trait which then affects decisionmaking behavior toward more patient versus more impulsive intertemporal choices. Our research suggests that the Val allele predisposes individuals to a low level of baseline activation in the 
left DPFC which then biases them toward impatient choice. These insights are highly relevant for a better understanding of potential risk factors and predispositions for suboptimal behaviors related to impulsivity and self-control failure and may suggest new treatment and intervention approaches for such disorders. For example, from a therapeutic point of view, our results may advance the development of individually tailored focal neuro-feedback treatment or personalized application of non-invasive brain stimulation to alter the level of cortical activation in certain brain areas. At a more general level, this individual differences approach, which explains individuals' variance in DD by an objective assessment of stable neuro-genetic markers, can set new avenues for future studies on

\section{REFERENCES}

Anokhin, A. P., Golosheykin, S., Grant, J. D., and Heath, A. C. (2011). Heritability of delay discounting in adolescence: a longitudinal twin study. Behav. Genet. 41, 175-183.

Beck, R. C., and Triplett, M. F. (2009). Test-retest reliability of a groupadministered paper-pencil measure of delay discounting. Exp. Clin. Psychopharmacol. 17, 345-355.

Bickel, W. K., Miller, M. L., Yi, R., Kowal, B. P., Lindquist, D. M., and Pitcock, J. A. (2007). Behavioral and neuroeconomics of drug addiction: competing neural systems and temporal discounting processes. Drug Alcohol Depend. 90(Suppl. 1), S85-S91.

Bjork, J. M., Momenan, R., and Hommer, D. W. (2009). Delay discounting correlates with proportional lateral frontal cortex volumes. Biol. Psychiatry 65, 710-713.

Boettiger, C. A., Mitchell, J. M., Tavares, V. C., Robertson, M., Joslyn, G., D'esposito, M., and Fields, H. L. (2007). Immediate reward bias in humans: frontoparietal networks and a role for the catechol-O-methyltransferase 158(Val/Val) genotype. J. Neurosci. 27, 14383-14391.

Bonnet, M. H., and Arand, D. L. (2001). Impact of activity and arousal upon spectral EEG parameters. Physiol. Behav. 74, 291-298.

Bouyer, J. J., Montaron, M. F., Vahnee, J. M., Albert, M. P., and Rougeul, A. (1987). Anatomical localization of cortical beta rhythms in cat. Neuroscience 22, 863-869.

Braver, T. S., Cole, M. W., and Yarkoni, T. (2011). Vive les differences! Individual variation in neural mechanisms of executive control. Curr. Opin. Neurobiol. 20, 242-250.

Carter, R. M., Meyer, J. R., and Huettel, S. A. (2010). Functional neuroimaging of intertemporal choice models: a review. J. Neurosci. Psychol. Econ. 3, 27-45.

Casey, B. J., Somerville, L. H., Gotlib, I. H., Ayduk, O., Franklin, N. T., Askren, M. K., Jonides, J., Berman, M. G., Wilson, N. L., Teslovich, T. Glover, G., Zayas, V., Mischel, W., and Shoda, Y. (2011). Behavioral and neural correlates of delay of gratification 40 years later. Proc. Natl. Acad. Sci. U.S.A. 108, 14988-15003.

Chen, J., Lipska, B. K., Halim, N., Ma, Q. D., Matsumoto, M., Melhem, S., Kolachana, B. S., Hyde, T. M., Herman, M. M., Apud, J., Egan, M. F., Kleinman, J. E., and Weinberger, D. R. (2004). Functional analysis of genetic variation in catechol- $O$ methyltransferase (COMT): effects on mRNA, protein, and enzyme activity in postmortem human brain. Am. J. Hum. Genet. 75, 807-821.

De Geus, E. J. (2010). From genotype to EEG endophenotype: a route for post-genomic understanding of complex psychiatric disease? Genome Med. 2, 63.

Dunki, R. M., Schmid, G. B., and Stassen, H. H. (2000). Intraindividual specificity and stability of human EEG: comparing a linear vs a nonlinear approach. Methods Inf. Med. 39, 78-82.

Figner, B., Knoch, D., Johnson, E. J., Krosch, A. R., Lisanby, S. H., Fehr, E., and Weber, E. U. (2010). Lateral prefrontal cortex and self-control in intertemporal choice. Nat. Neurosci. 13, 538-539.

Gamma, A., Lehmann, D., Frei, E., Iwata, K., Pascual-Marqui, R. D., and Vollenweider, F. X. (2004). Comparison of simultaneously recorded [H2(15)O]-PET and LORETA during cognitive and pharmacological activation. Hum. Brain Mapp. 22, 83-96.

Gianotti, L. R., Knoch, D., Faber, P. L., Lehmann, D., Pascual-Marqui, R. D., Diezi, C., Schoch, C., Eisenegger, C., and Fehr, E. (2009). Tonic activity

individual differences in behaviors and personality traits in other domains.

\section{ACKNOWLEDGMENTS}

This work was supported by a grant from the Swiss National Science Foundation to Daria Knoch (PP00P1-123381). Bernd Figner acknowledges support from the Swiss National Science Foundation (grant PA001-15327) and from the U.S. National Science Foundation (grant SES-0922743). We thank T. E. Gladwin, D. J. Hardisty, L. Jäncke, E. J. Johnson, D. Lehmann, R. D. PascualMarqui, and E. U. Weber. We also thank O. Hager, N. Langer, N. Mock, and I. Weidmann for their help in collecting the data.

level in the right prefrontal cortex predicts individuals' risk taking. Psychol. Sci. 20, 33-38.

Green, L., and Myerson, J. (2004). A discounting framework for choice with delayed and probabilistic rewards. Psychol. Bull. 130, 769-792.

Hackman, D. A., Farah, M. J., and Meaney, M. J. (2010). Socioeconomic status and the brain: mechanistic insights from human and animal research. Nat. Rev. Neurosci. 11 , 651-659.

Hare, T. A., Camerer, C. F., and Rangel, A. (2009). Self-control in decisionmaking involves modulation of the vmPFC valuation system. Science 324, 646-648.

Kirby, K. N. (2009). One-year temporal stability of delay-discount rates. Psychon. Bull. Rev. 16, 457-462.

Knoch, D., Gianotti, L. R., Baumgartner, T., and Fehr, E. (2010). A neural marker of costly punishment behavior. Psychol. Sci. 21, 337-342.

Kubicki, S., Herrmann, W. M., Fichte, K., and Freund, G. (1979). Reflections on the topics: EEG frequency bands and regulation of vigilance. Pharmakopsychiatr. Neuropsychopharmakol. 12, 237-245.

Laufs, H., Krakow, K., Sterzer, P., Eger, E., Beyerle, A., Salek-Haddadi, A., and Kleinschmidt, A. (2003). Electroencephalographic signatures of attentional and cognitive default modes in spontaneous brain activity fluctuations at rest. Proc. Natl. Acad. Sci. U.S.A. 100, 11053-11058.

Liu, B., Song, M., Li, J., Liu, Y., Li, K., Yu, C., and Jiang, T. (2010). Prefrontal-related functional connectivities within the default network are modulated by COMT val158met in healthy young adults. J. Neurosci. 30, 64-69.

MacKillop, J., Amlung, M. T., Few, L. R., Ray, L. A., Sweet, L. H., and Munafo, M. R. (2011). Delayed reward discounting and addictive behavior: a meta-analysis. Psychopharmacology (Berl.) 216, 305-321.
MacKinnon, D. P., Fairchild, A. J., and Fritz, M. S. (2007). Mediation analysis. Annu. Rev. Psychol. 58, 593-614.

McClure, S. M., Laibson, D. I., Loewenstein, G., and Cohen, J. D. (2004). Separate neural systems value immediate and delayed monetary rewards. Science 306, 503-507.

Meyer-Lindenberg, A., and Weinberger, D. R. (2006). Intermediate phenotypes and genetic mechanisms of psychiatric disorders. Nat. Rev. Neurosci. 7, 818-827.

Mischel, W., Ayduk, O., Berman, M. G., Casey, B. J., Gotlib, I. H., Jonides, J., Kross, E., Teslovich, T., Wilson, N. L., Zayas, V., and Shoda, Y. (2011). 'Willpower' over the life span: decomposing selfregulation. Soc. Cogn. Affect. Neurosci. 6, 252-256.

Monterosso, J., and Ainslie, G. (1999). Beyond discounting: possible experimental models of impulse control. Psychopharmacology (Berl.) 146, 339-347.

Murthy, V. N., and Fetz, E. E. (1992). Coherent $25-$ to $35-\mathrm{Hz}$ oscillations in the sensorimotor cortex of awake behaving monkeys. Proc. Natl. Acad. Sci. U.S.A. 89, 5670-5674.

Napflin, M., Wildi, M., and Sarnthein, J. (2007). Test-retest reliability of resting EEG spectra validates a statistical signature of persons. Clin. Neurophysiol. 118, 2519-2524.

Nichols, T. E., and Holmes, A. P. (2002). Nonparametric permutation tests for functional neuroimaging: a primer with examples. Hum. Brain Mapp. 15, 1-25.

Oakes, T. R., Pizzagalli, D. A., Hendrick, A. M., Horras, K. A., Larson, C. L., Abercrombie, H. C., Schaefer, S. M., Koger, J. V., and Davidson, R. J. (2004). Functional coupling of simultaneous electrical and metabolic activity in the human brain Hum. Brain Mapp. 21, 257-270.

Odum, A. L. (2011). Delay discounting: trait variable? Behav. Processes $87,1-9$. 
Olson, E. A., Collins, P. F., Hooper, C. J., Muetzel, R., Lim, K. O., and Luciana, M. (2009). White matter integrity predicts delay discounting behavior in 9- to 23-year-olds: a diffusion tensor imaging study. J. Cogn. Neurosci. 21, 1406-1421.

Paloyelis, Y., Asherson, P., Mehta, M. A., Faraone, S. V., and Kuntsi, J. (2010). DAT1 and COMT effects on delay discounting and trait impulsivity in male adolescents with attention deficit/hyperactivity disorder and healthy controls. Neuropsychopharmacology 35, 2414-2426.

Pascual-Marqui, R. D. (2002). Standardized low-resolution brain electromagnetic tomography (sLORETA): technical details. Methods Find Exp. Clin. Pharmacol. 24(Suppl. D), 5-12.

Patton, J. H., Stanford, M. S., and Barratt, E. S. (1995). Factor structure of the Barratt impulsiveness scale. J. Clin. Psychol. 51, 768-774.

Paus, T., Sipila, P. K., and Strafella, A. P. (2001). Synchronization of neuronal activity in the human primary motor cortex by transcranial magnetic stimulation: an EEG study. J. Neurophysiol. 86, 1983-1990.

Peters, J., and Buchel, C. (2010). Episodic future thinking reduces reward delay discounting through an enhancement of prefrontalmediotemporal interactions. $\mathrm{Neu}$ ron 66, 138-148.

Peters, J., and Buchel, C. (2011). The neural mechanisms of inter-temporal decision-making: understanding variability. Trends Cogn. Sci. (Regul. Ed.) 15, 227-239.

Pizzagalli, D. A., Sherwood, R. J., Henriques, J. B., and Davidson, R. J. (2005). Frontal brain asymmetry and reward responsiveness: a sourcelocalization study. Psychol. Sci. 16, 805-813.

Preacher, K. J., and Hayes, A. F. (2008). Asymptotic and resampling strategies for assessing and comparing indirect effects in multiple mediator models. Behav. Res. Methods 40, 879-891.

Raftery, A. E. (1995). Bayesian model selection in social research (with Discussion). Sociol. Methodol. 25, 111-196.

Reimers, S., Maylor, E. A., Stewart, N., and Chater, N. (2009). Association between a one-shot delay discounting measure and age, income education and real-world impulsive behavior. Pers. Individ. Dif. 47, 973-978.

Reynolds, B. (2006). A review of delaydiscounting research with humans: relations to drug use and gambling. Behav. Pharmacol. 17, 651-667.

Scheres, A., Tontsch, C., Thoeny, A. L., and Kaczkurkin, A. (2010). Temporal reward discounting in attention-deficit/hyperactivity disorder: the contribution of symptom domains, reward magnitude, and session length. Biol. Psychiatry 67, 641-648.

Shamosh, N. A., Deyoung, C. G., Green, A. E., Reis, D. L., Johnson, M. R.,
Conway, A. R., Engle, R. W., Braver, T. S., and Gray, J. R. (2008). Individual differences in delay discounting: relation to intelligence, working memory, and anterior prefrontal cortex. Psychol. Sci. 19, 904-911.

Smit, D. J., Posthuma, D., Boomsma, D. I., and Geus, E. J. (2005). Heritability of background EEG across the power spectrum. Psychophysiology 42, 691-697.

Tunbridge, E. M., Bannerman, D. M., Sharp, T., and Harrison, P. J. (2004). Catechol-o-methyltransferase inhibition improves set-shifting performance and elevates stimulated dopamine release in the rat prefrontal cortex. J. Neurosci. 24, 5331-5335.

van 't Ent, D., Van Soelen, I. L., Stam, C. J., De Geus, E. J., and Boomsma, D. I. (2009). Strong resemblance in the amplitude of oscillatory brain activity in monozygotic twins is not caused by "trivial" similarities in the composition of the skull. Hum. Brain Mapp. 30, 2142-2145.

Wager, T. D., Davidson, M. L., Hughes, B. L., Lindquist, M. A., and Ochsner, K. N. (2008). Prefrontalsubcortical pathways mediating successful emotion regulation. Neuron 59, 1037-1050.

Watson, D., Clark, L. A., and Tellegen, A. (1988). Development and validation of brief measures of positive and negative affect: the PANAS scales. $J$. Pers. Soc. Psychol. 54, 1063-1070.

Winstanley, C. A., Eagle, D. M., and Robbins, T. W. (2006). Behavioral models of impulsivity in relation to ADHD: translation between clinical and preclinical studies. Clin. Psychol. Rev. 26, 379-395.

Zietsch, B. P., Hansen, J. L., Hansell, N. K., Geffen, G. M., Martin, N. G., and Wright, M. J. (2007). Common and specific genetic influences on EEG power bands delta, theta, alpha, and beta. Biol. Psychol. 75, 154-164.

Conflict of Interest Statement: The authors declare that the research was conducted in the absence of any commercial or financial relationships that could be construed as a potential conflict of interest.

Received: 09 January 2012; accepted: 29 March 2012; published online: 07 May 2012.

Citation: Gianotti LRR, Figner B, Ebstein $R P$ and Knoch D (2012) Why some people discount more than others: baseline activation in the dorsal PFC mediates the link between COMT genotype and impatient choice. Front. Neurosci. 6:54. doi 10.3389/fnins.2012.00054

This article was submitted to Frontiers in Decision Neuroscience, a specialty of Frontiers in Neuroscience.

Copyright (C) 2012 Gianotti, Figner, Ebstein and Knoch. This is an open-access article distributed under the terms of the Creative Commons Attribution Non Commercial License, which permits noncommercial use, distribution, and reproduction in other forums, provided the original authors and source are credited. 


\section{APPENDIX \\ METHODS \\ Intertemporal choice task}

In the intertemporal choice task, participants made 36 binary choices, each between a sooner-smaller reward (SS) and a laterlarger (LL) reward. The 36 trials represented a full factorial design that varied (i) the time of delivery of SS, (ii) the time interval between SS and LL, and (iii) the relative difference in reward magnitudes of SS and LL. In 18 trials, the time of delivery of the SS was "today" while in the other 18 trials the time of delivery of the SS was "in 2 weeks." Crossed with this, the time interval between SS and LL was either 2 or 4 weeks. Crossed with the two previous factors, the relative difference in reward magnitudes of SS and LL was varied, with the LL being $0.5,1,5,10,15,20,25,30,50$, or $75 \%$ larger than the SS (the task was based on the original task used by McClure et al. (2004), but adjusted for our study; see also Figner et al. (2010)).

Amounts for the SS were pseudo-randomly drawn from a normal distribution with a mean of Swiss Francs (CHF) 45, capped at the lower end at CHF 15 and CHF 85 at the higher end. On each trial, participants were presented simultaneously with the SS and the LL on a computer screen (SS was always presented on the left) and expressed their choice by clicking on the radio button below their preferred option. They started the next trial by clicking on a button placed in the middle of the screen. The task was incentivecompatible, i.e., as explained to participants at the beginning of the study, one choice of all the choices made was randomly selected at the end of the study, and the chosen option on this trial was paid out for real, i.e., the chosen amount was transferred to their bank account on the chosen day of delivery.

The overwhelming majority of the literature reports better fits for hyperbolic than exponential discounting models (for an overview, see Green and Myerson (2004)). Accordingly, we used Mazur's standard one-parameter model of hyperbolic discounting (Mazur, 1987) with the discount rate $k$ as individual differences indicator for the steepness of delay discounting.

Subjective Value $=\frac{\text { Objective Amount }}{1+k \times \text { Delay }}$

Delay indicates the time of delivery (in years) and $k$ is a constant that is specific to each participant and indicates the steepness of the participant's discounting (with larger values indicating steeper discounting and 0 indicating no discounting at all).

For each participant, the hyperbolic discount rate $k$ best explaining their choice pattern was estimated by fitting a logistic curve to their choices (plotting choice of SS versus LL as a function of the indifference-implied discount rate of each choice pair). The participant's estimated discount rate was thus the discount rate at which the participant would be predicted to choose the SS and LL with equal probability. Discount rates were square-root transformed for use in further analysis.

\section{DNA preparation and genotyping}

Participants were instructed to rinse their mouths with $25 \mathrm{ml}$ of mouthwash and to spit the fluid into sterile $50-\mathrm{ml}$ polypropylene tubes. The samples were stored at $4^{\circ} \mathrm{C}$ if they could not be processed within $2 \mathrm{~h}$ of collection. DNA from these samples was extracted and amplified (Genetica, Zurich, Switzerland).

The following procedure was performed: the tubes were centrifuged at 1600 relative centrifugal force/g using a Hettich Rotina 46 S centrifuge (Hettich AG Laborapparate, Baech, Switzerland). The remaining pellet was resuspended in $180 \mu \mathrm{l}$ Tissue Lysis Buffer (Qiagen AG, Hombrechtikon, Switzerland) and proteinase $\mathrm{K}$ was added $(30 \mu \mathrm{l}$ of a $20-\mathrm{mg} / \mathrm{ml}$ stock solution). This solution was hybridized for $3 \mathrm{~h}$ at $58^{\circ} \mathrm{C}$. The solution was then stirred and transferred into a 2-ml test tube. The tube was centrifuged for $1 \mathrm{~min}$ at $10,000 \mathrm{rpm}$. A standard EZ1 DNA extraction was performed from this mixture using the BioRobot EZ1 following the QIAamp Blood Kit Protocol (obtained from Qiagen AG). The obtained DNA concentration was then measured using a photometer (Nanodrop, Fisher Scientific, GmbH, Schwerte, Germany). The Val158Met polymorphism of the COMT gene was characterized using the SNaPshot Method (Applied BioSystems, Foster City, CA, USA). This method relies upon the extension of a primer immediately adjacent to the SNP using fluorescently labeled ddNTPs. The fluorescently labeled extension primers can then be visualized by electrophoresis on a capillary ABI PRISM 310 automated sequencer. The COMT SNP region was amplified using the following primers:

\section{First PCR primers: F5' -ATCGGGCTACTCAGCTGTG-3'}

R5'-CCTTTTTCCAGGTCTGACAA- ${ }^{\prime}$

Second PCR primer extension: $5^{\prime}$-TGAAGGACAAGGTGTG CATG-3'

PCR cycling conditions in the SNaPshot Method were as follows: samples were initially heated at $94^{\circ} \mathrm{C}$ for $5 \mathrm{~min}$ followed by 35 cycles of $94^{\circ} \mathrm{C}(30 \mathrm{~s}), 55^{\circ} \mathrm{C}(30 \mathrm{~s}), 72^{\circ} \mathrm{C}(90 \mathrm{~s})$, and a final extension step of $72^{\circ} \mathrm{C}$ for $5 \mathrm{~min}$. After the first PCR cycle, the PCR product was cleaned with ExoSAP for $37^{\circ} \mathrm{C}$ for $30 \mathrm{~min}$ and then at $80^{\circ} \mathrm{C}$ for $15 \mathrm{~min}$. The conditions for the second PCR were as follows: $96^{\circ} \mathrm{C}(10 \mathrm{~s}), 50^{\circ} \mathrm{C}(5 \mathrm{~s})$, and $60^{\circ} \mathrm{C}(30 \mathrm{~s})$ for 25 cycles. The second PCR product was cleaned using shrimp alkaline phosphatase (SAP) initially at $37^{\circ} \mathrm{C}$ for $1 \mathrm{~h}$ followed by $72^{\circ} \mathrm{C}$ for $15 \mathrm{~min}$.

\section{RESULTS}

Nineteen participants were homozygous for the Val allele, and 13 participants were homozygous for the Met allele of the COMT gene; 41 heterozygote participants carried one Val and one Met allele of the COMT gene. Genotype distribution was in Hardy-Weinberg equilibrium $\left(\chi^{2}=1.25, P=0.26\right)$.

Genotype groups did not differ in terms of age, educational level, levels of pre-task affect (PANAS), and impulsivity (BISII; Table A1), thus ruling out possible alternative explanations that relationships between COMT genotype, DD, and the baseline neural activity level were caused by differences in these variables. That BIS-II scores were not related to COMT genotype is consistent with other studies (e.g., Eisenegger et al., 2010) which also found a significant relationship between choices in a behavioral task and genes, but no significant relation with BIS-II. 
Table A1 | Means and SDs of demographic and individual differences variables characterizing the sample.

\begin{tabular}{|c|c|c|c|c|c|}
\hline & Val/Val & Val/Met & Met/Met & $F$-value & $P$-value \\
\hline$n$ & 19 & 41 & 13 & & \\
\hline Age (years) & $27.3(5.4)$ & $25.4(4.5)$ & $24.2(5.9)$ & 1.62 & 0.20 \\
\hline BIS-attentional score & $15.7(3.2)$ & $15.6(3.3)$ & $15.6(2.4)$ & 0.02 & 0.98 \\
\hline BIS-motor score & $22.5(3.6)$ & $23.9(4.4)$ & $24.1(4.5)$ & 0.81 & 0.45 \\
\hline PANAS PA_trait & $33.1(4.3)$ & $33.5(4.6)$ & $33.3(5.7)$ & 0.04 & 0.96 \\
\hline PANAS NA_trait & $17.4(5.1)$ & $16.0(3.9)$ & $16.1(2.4)$ & 0.86 & 0.43 \\
\hline PANAS PA_state & $29.3(4.5)$ & $30.2(5.3)$ & $32.2(7.9)$ & 0.92 & 0.40 \\
\hline PANAS NA_state & $12.9(4.0)$ & $13.4(3.5)$ & $12.0(1.7)$ & 0.78 & 0.46 \\
\hline
\end{tabular}

BIS, Barratt Impulsivity Scale; PA_trait, Positive Affect Trait Scale; NA_trait, Negative Affect Trait scale; PA_state, Positive Affect State Scale; NA_state, Negative Affect State scale.

Table A2 | Spearman rank correlation coefficients for the short term test-retest EEG reliability.

\begin{tabular}{|c|c|c|c|c|c|c|c|c|c|c|c|c|c|c|c|}
\hline Channel & Delta & Theta & Alpha1 & Alpha2 & Beta1 & Beta2 & Beta3 & Channel & Delta & Theta & Alpha1 & Alpha2 & Beta1 & Beta2 & Beta3 \\
\hline 2 & 0.96 & 0.97 & 0.97 & 0.96 & 0.96 & 0.95 & 0.96 & 64 & 0.98 & 0.98 & 0.97 & 0.98 & 0.99 & 0.98 & 0.99 \\
\hline 4 & 0.97 & 0.96 & 0.97 & 0.95 & 0.96 & 0.95 & 0.97 & 66 & 0.99 & 0.98 & 0.98 & 0.98 & 0.99 & 0.98 & 0.99 \\
\hline 5 & 0.97 & 0.97 & 0.97 & 0.97 & 0.97 & 0.96 & 0.98 & 67 & 0.98 & 0.98 & 0.97 & 0.97 & 0.98 & 0.98 & 0.99 \\
\hline 6 & 0.97 & 0.96 & 0.98 & 0.95 & 0.97 & 0.97 & 0.98 & 69 & 0.99 & 0.98 & 0.97 & 0.98 & 0.99 & 0.98 & 0.99 \\
\hline 8 & 0.98 & 0.97 & 0.94 & 0.97 & 0.97 & 0.96 & 0.97 & 71 & 0.99 & 0.99 & 0.98 & 0.98 & 0.99 & 0.98 & 0.99 \\
\hline 9 & 0.97 & 0.97 & 0.96 & 0.97 & 0.98 & 0.96 & 0.97 & 72 & 0.99 & 0.99 & 0.97 & 0.97 & 0.99 & 0.98 & 0.99 \\
\hline 10 & 0.96 & 0.98 & 0.97 & 0.97 & 0.97 & 0.98 & 0.98 & 74 & 0.99 & 0.98 & 0.97 & 0.95 & 0.99 & 0.99 & 0.99 \\
\hline 11 & 0.96 & 0.96 & 0.97 & 0.97 & 0.98 & 0.96 & 0.98 & 75 & 0.99 & 0.99 & 0.97 & 0.98 & 0.99 & 0.99 & 0.99 \\
\hline 12 & 0.97 & 0.97 & 0.95 & 0.96 & 0.98 & 0.96 & 0.97 & 76 & 0.99 & 0.98 & 0.98 & 0.98 & 0.98 & 0.99 & 0.99 \\
\hline 13 & 0.96 & 0.96 & 0.94 & 0.97 & 0.96 & 0.96 & 0.97 & 77 & 0.98 & 0.98 & 0.97 & 0.97 & 0.99 & 0.98 & 0.99 \\
\hline 17 & 0.98 & 0.96 & 0.97 & 0.96 & 0.98 & 0.97 & 0.97 & 82 & 0.99 & 0.98 & 0.98 & 0.98 & 0.99 & 0.98 & 0.99 \\
\hline 18 & 0.99 & 0.98 & 0.98 & 0.98 & 0.99 & 0.98 & 0.98 & 83 & 0.99 & 0.97 & 0.97 & 0.98 & 0.99 & 0.98 & 0.99 \\
\hline 19 & 0.96 & 0.96 & 0.97 & 0.98 & 0.98 & 0.97 & 0.98 & 84 & 0.98 & 0.98 & 0.97 & 0.97 & 0.99 & 0.98 & 0.99 \\
\hline 20 & 0.97 & 0.97 & 0.96 & 0.97 & 0.97 & 0.98 & 0.97 & 85 & 0.98 & 0.98 & 0.97 & 0.97 & 0.98 & 0.98 & 0.99 \\
\hline 21 & 0.95 & 0.98 & 0.97 & 0.98 & 0.98 & 0.97 & 0.98 & 86 & 0.98 & 0.97 & 0.97 & 0.99 & 0.99 & 0.98 & 0.99 \\
\hline 22 & 0.98 & 0.97 & 0.96 & 0.98 & 0.98 & 0.98 & 0.97 & 87 & 0.98 & 0.98 & 0.97 & 0.98 & 0.99 & 0.98 & 0.99 \\
\hline 23 & 0.98 & 0.98 & 0.97 & 0.97 & 0.99 & 0.97 & 0.98 & 89 & 0.99 & 0.98 & 0.98 & 0.99 & 0.99 & 0.98 & 0.99 \\
\hline 24 & 0.97 & 0.95 & 0.95 & 0.97 & 0.96 & 0.97 & 0.96 & 90 & 0.99 & 0.98 & 0.98 & 0.99 & 0.99 & 0.99 & 0.99 \\
\hline 25 & 0.97 & 0.94 & 0.97 & 0.96 & 0.97 & 0.96 & 0.97 & 91 & 0.98 & 0.97 & 0.96 & 0.98 & 0.99 & 0.98 & 0.98 \\
\hline 26 & 0.98 & 0.98 & 0.97 & 0.98 & 0.98 & 0.98 & 0.99 & 92 & 0.98 & 0.97 & 0.97 & 0.98 & 0.98 & 0.98 & 0.98 \\
\hline 27 & 0.98 & 0.96 & 0.96 & 0.97 & 0.98 & 0.96 & 0.99 & 93 & 0.99 & 0.98 & 0.97 & 0.97 & 0.98 & 0.98 & 0.99 \\
\hline
\end{tabular}


Table A2 | Continued

\begin{tabular}{|c|c|c|c|c|c|c|c|c|c|c|c|c|c|c|c|}
\hline Channel & Delta & Theta & Alpha1 & Alpha2 & Beta1 & Beta2 & Beta3 & Channel & Delta & Theta & Alpha1 & Alpha2 & Beta1 & Beta2 & Beta3 \\
\hline 33 & 0.99 & 0.97 & 0.97 & 0.97 & 0.99 & 0.98 & 0.98 & 101 & 0.99 & 0.97 & 0.97 & 0.98 & 0.98 & 0.97 & 0.97 \\
\hline 34 & 0.99 & 0.98 & 0.97 & 0.98 & 0.98 & 0.98 & 0.99 & 102 & 0.98 & 0.98 & 0.97 & 0.98 & 0.98 & 0.98 & 0.98 \\
\hline 35 & 0.98 & 0.97 & 0.97 & 0.97 & 0.98 & 0.98 & 0.98 & 103 & 0.99 & 0.98 & 0.96 & 0.97 & 0.98 & 0.97 & 0.98 \\
\hline 36 & 0.98 & 0.97 & 0.96 & 0.97 & 0.97 & 0.97 & 0.97 & 104 & 0.98 & 0.98 & 0.96 & 0.97 & 0.98 & 0.96 & 0.97 \\
\hline 37 & 0.97 & 0.98 & 0.97 & 0.97 & 0.99 & 0.98 & 0.98 & 105 & 0.98 & 0.97 & 0.93 & 0.97 & 0.98 & 0.98 & 0.98 \\
\hline 38 & 0.96 & 0.96 & 0.96 & 0.97 & 0.98 & 0.96 & 0.98 & 106 & 0.99 & 0.97 & 0.97 & 0.97 & 0.98 & 0.98 & 0.95 \\
\hline 39 & 0.98 & 0.98 & 0.98 & 0.97 & 0.97 & 0.96 & 0.97 & 108 & 0.99 & 0.97 & 0.97 & 0.97 & 0.99 & 0.98 & 0.99 \\
\hline 40 & 0.98 & 0.98 & 0.97 & 0.98 & 0.98 & 0.97 & 0.98 & 109 & 0.99 & 0.97 & 0.96 & 0.97 & 0.99 & 0.98 & 0.98 \\
\hline 41 & 0.99 & 0.96 & 0.96 & 0.95 & 0.98 & 0.97 & 0.99 & 110 & 0.99 & 0.98 & 0.97 & 0.97 & 0.99 & 0.97 & 0.98 \\
\hline 42 & 0.98 & 0.96 & 0.94 & 0.96 & 0.98 & 0.98 & 0.99 & 111 & 0.98 & 0.98 & 0.96 & 0.98 & 0.99 & 0.98 & 0.98 \\
\hline 43 & 0.98 & 0.98 & 0.97 & 0.97 & 0.99 & 0.98 & 0.99 & 112 & 0.99 & 0.98 & 0.96 & 0.98 & 0.98 & 0.98 & 0.98 \\
\hline 44 & 0.99 & 0.97 & 0.97 & 0.97 & 0.99 & 0.97 & 0.98 & 114 & 0.99 & 0.98 & 0.96 & 0.97 & 0.99 & 0.97 & 0.98 \\
\hline 45 & 0.98 & 0.97 & 0.97 & 0.97 & 0.98 & 0.97 & 0.99 & 115 & 0.98 & 0.97 & 0.96 & 0.98 & 0.99 & 0.98 & 0.99 \\
\hline 46 & 0.98 & 0.98 & 0.98 & 0.97 & 0.99 & 0.98 & 0.99 & 116 & 0.98 & 0.99 & 0.97 & 0.99 & 0.99 & 0.99 & 0.98 \\
\hline 47 & 0.99 & 0.97 & 0.97 & 0.97 & 0.99 & 0.97 & 0.99 & 117 & 0.98 & 0.97 & 0.97 & 0.97 & 0.98 & 0.98 & 0.98 \\
\hline 50 & 0.99 & 0.98 & 0.97 & 0.97 & 0.99 & 0.99 & 0.99 & 118 & 0.97 & 0.98 & 0.98 & 0.98 & 0.98 & 0.98 & 0.97 \\
\hline 51 & 0.99 & 0.97 & 0.97 & 0.97 & 0.99 & 0.99 & 0.99 & 120 & 0.98 & 0.96 & 0.98 & 0.99 & 0.99 & 0.98 & 0.99 \\
\hline 52 & 0.99 & 0.98 & 0.98 & 0.98 & 0.99 & 0.98 & 0.99 & 121 & 0.99 & 0.98 & 0.98 & 0.98 & 0.98 & 0.99 & 0.99 \\
\hline 53 & 0.98 & 0.98 & 0.97 & 0.98 & 0.98 & 0.98 & 0.98 & 122 & 0.99 & 0.99 & 0.98 & 0.98 & 0.99 & 0.98 & 0.98 \\
\hline 54 & 0.98 & 0.98 & 0.97 & 0.96 & 0.98 & 0.98 & 0.98 & 123 & 0.99 & 0.99 & 0.98 & 0.98 & 0.97 & 0.98 & 0.98 \\
\hline 55 & 0.99 & 0.98 & 0.98 & 0.98 & 0.99 & 0.98 & 0.98 & 124 & 0.99 & 0.99 & 0.98 & 0.98 & 0.99 & 0.97 & 0.98 \\
\hline 57 & 0.99 & 0.98 & 0.98 & 0.98 & 0.99 & 0.98 & 0.98 & & & & & & & & \\
\hline 58 & 0.99 & 0.98 & 0.97 & 0.98 & 0.99 & 0.99 & 0.99 & & & & & & & & \\
\hline 59 & 0.99 & 0.97 & 0.97 & 0.97 & 0.98 & 0.99 & 0.99 & Min & 0.95 & 0.94 & 0.93 & 0.95 & 0.96 & 0.95 & 0.95 \\
\hline 60 & 0.99 & 0.98 & 0.97 & 0.97 & 0.98 & 0.99 & 0.99 & Max & 0.99 & 0.99 & 0.98 & 0.99 & 0.99 & 0.99 & 0.99 \\
\hline 61 & 0.98 & 0.97 & 0.97 & 0.97 & 0.97 & 0.97 & 0.98 & Mean & 0.98 & 0.97 & 0.97 & 0.97 & 0.98 & 0.98 & 0.98 \\
\hline
\end{tabular}

Absolute power values were compared between the first and the second half of the recorded data.

Table A3 | Spearman rank correlation coefficients for the short term test-retest EEG reliability.

\begin{tabular}{|c|c|c|c|c|c|c|c|c|c|c|c|c|c|c|c|}
\hline Channel & Delta & Theta & Alpha1 & Alpha2 & Beta1 & Beta2 & Beta3 & Channel & Delta & Theta & Alpha1 & Alpha2 & Beta1 & Beta2 & Beta3 \\
\hline 2 & 0.91 & 0.95 & 0.91 & 0.91 & 0.90 & 0.88 & 0.91 & 64 & 0.95 & 0.95 & 0.94 & 0.96 & 0.97 & 0.95 & 0.96 \\
\hline 4 & 0.90 & 0.93 & 0.89 & 0.89 & 0.89 & 0.88 & 0.91 & 66 & 0.97 & 0.95 & 0.94 & 0.94 & 0.97 & 0.94 & 0.96 \\
\hline 5 & 0.94 & 0.94 & 0.93 & 0.92 & 0.92 & 0.90 & 0.95 & 67 & 0.97 & 0.96 & 0.94 & 0.92 & 0.96 & 0.92 & 0.96 \\
\hline 6 & 0.92 & 0.92 & 0.93 & 0.90 & 0.93 & 0.90 & 0.94 & 69 & 0.96 & 0.96 & 0.94 & 0.95 & 0.96 & 0.97 & 0.97 \\
\hline 8 & 0.94 & 0.95 & 0.86 & 0.92 & 0.90 & 0.89 & 0.90 & 71 & 0.98 & 0.98 & 0.96 & 0.95 & 0.98 & 0.95 & 0.97 \\
\hline 9 & 0.93 & 0.94 & 0.90 & 0.90 & 0.90 & 0.86 & 0.92 & 72 & 0.98 & 0.97 & 0.95 & 0.93 & 0.96 & 0.95 & 0.97 \\
\hline 10 & 0.93 & 0.94 & 0.92 & 0.93 & 0.93 & 0.92 & 0.97 & 74 & 0.98 & 0.97 & 0.93 & 0.90 & 0.97 & 0.94 & 0.97 \\
\hline 11 & 0.90 & 0.92 & 0.91 & 0.92 & 0.93 & 0.88 & 0.94 & 75 & 0.97 & 0.96 & 0.95 & 0.94 & 0.97 & 0.95 & 0.97 \\
\hline 12 & 0.91 & 0.92 & 0.86 & 0.88 & 0.92 & 0.88 & 0.91 & 76 & 0.98 & 0.95 & 0.94 & 0.94 & 0.97 & 0.96 & 0.98 \\
\hline 13 & 0.91 & 0.93 & 0.88 & 0.89 & 0.89 & 0.88 & 0.93 & 77 & 0.97 & 0.97 & 0.94 & 0.92 & 0.96 & 0.94 & 0.96 \\
\hline 17 & 0.96 & 0.95 & 0.92 & 0.90 & 0.93 & 0.92 & 0.95 & 82 & 0.97 & 0.97 & 0.95 & 0.96 & 0.97 & 0.95 & 0.96 \\
\hline
\end{tabular}


Table A3 | Continued

\begin{tabular}{|c|c|c|c|c|c|c|c|c|c|c|c|c|c|c|c|}
\hline Channel & Delta & Theta & Alpha1 & Alpha2 & Beta1 & Beta2 & Beta3 & Channel & Delta & Theta & Alpha1 & Alpha2 & Beta1 & Beta2 & Beta3 \\
\hline 18 & 0.96 & 0.95 & 0.93 & 0.91 & 0.95 & 0.95 & 0.96 & 83 & 0.97 & 0.94 & 0.94 & 0.96 & 0.96 & 0.95 & 0.97 \\
\hline 19 & 0.93 & 0.93 & 0.93 & 0.94 & 0.95 & 0.92 & 0.96 & 84 & 0.96 & 0.96 & 0.94 & 0.94 & 0.97 & 0.95 & 0.97 \\
\hline 20 & 0.93 & 0.93 & 0.89 & 0.89 & 0.90 & 0.90 & 0.93 & 85 & 0.96 & 0.96 & 0.93 & 0.92 & 0.94 & 0.92 & 0.95 \\
\hline 21 & 0.92 & 0.93 & 0.91 & 0.94 & 0.94 & 0.92 & 0.95 & 86 & 0.97 & 0.96 & 0.92 & 0.95 & 0.97 & 0.96 & 0.96 \\
\hline 22 & 0.95 & 0.94 & 0.90 & 0.92 & 0.95 & 0.91 & 0.93 & 87 & 0.96 & 0.97 & 0.92 & 0.95 & 0.97 & 0.95 & 0.98 \\
\hline 23 & 0.96 & 0.95 & 0.93 & 0.94 & 0.96 & 0.91 & 0.97 & 89 & 0.98 & 0.96 & 0.93 & 0.95 & 0.97 & 0.96 & 0.97 \\
\hline 24 & 0.95 & 0.91 & 0.91 & 0.91 & 0.90 & 0.87 & 0.90 & 90 & 0.98 & 0.97 & 0.93 & 0.96 & 0.97 & 0.96 & 0.97 \\
\hline 25 & 0.92 & 0.91 & 0.92 & 0.89 & 0.92 & 0.89 & 0.92 & 91 & 0.94 & 0.95 & 0.91 & 0.94 & 0.96 & 0.93 & 0.96 \\
\hline 26 & 0.94 & 0.96 & 0.94 & 0.90 & 0.96 & 0.95 & 0.96 & 92 & 0.97 & 0.95 & 0.94 & 0.94 & 0.95 & 0.95 & 0.96 \\
\hline 27 & 0.94 & 0.93 & 0.91 & 0.91 & 0.92 & 0.88 & 0.94 & 93 & 0.95 & 0.95 & 0.93 & 0.92 & 0.95 & 0.94 & 0.96 \\
\hline 28 & 0.95 & 0.90 & 0.91 & 0.89 & 0.93 & 0.90 & 0.96 & 95 & 0.98 & 0.96 & 0.94 & 0.93 & 0.95 & 0.95 & 0.96 \\
\hline 29 & 0.93 & 0.92 & 0.93 & 0.92 & 0.92 & 0.87 & 0.94 & 96 & 0.98 & 0.96 & 0.95 & 0.96 & 0.98 & 0.96 & 0.97 \\
\hline 30 & 0.89 & 0.91 & 0.90 & 0.92 & 0.93 & 0.93 & 0.93 & 97 & 0.98 & 0.96 & 0.94 & 0.93 & 0.96 & 0.95 & 0.96 \\
\hline 31 & 0.96 & 0.94 & 0.95 & 0.91 & 0.95 & 0.91 & 0.93 & 98 & 0.97 & 0.98 & 0.93 & 0.95 & 0.96 & 0.93 & 0.96 \\
\hline 32 & 0.96 & 0.91 & 0.92 & 0.90 & 0.94 & 0.93 & 0.95 & 100 & 0.97 & 0.96 & 0.91 & 0.95 & 0.97 & 0.94 & 0.95 \\
\hline 33 & 0.97 & 0.95 & 0.94 & 0.91 & 0.97 & 0.96 & 0.96 & 101 & 0.96 & 0.95 & 0.93 & 0.93 & 0.95 & 0.91 & 0.93 \\
\hline 34 & 0.98 & 0.93 & 0.94 & 0.94 & 0.96 & 0.93 & 0.96 & 102 & 0.97 & 0.96 & 0.92 & 0.96 & 0.95 & 0.93 & 0.96 \\
\hline 35 & 0.97 & 0.96 & 0.95 & 0.92 & 0.97 & 0.94 & 0.95 & 103 & 0.96 & 0.95 & 0.92 & 0.93 & 0.95 & 0.93 & 0.96 \\
\hline 36 & 0.95 & 0.93 & 0.91 & 0.92 & 0.94 & 0.92 & 0.94 & 104 & 0.94 & 0.94 & 0.91 & 0.91 & 0.93 & 0.88 & 0.93 \\
\hline 37 & 0.95 & 0.96 & 0.95 & 0.90 & 0.95 & 0.93 & 0.97 & 105 & 0.95 & 0.93 & 0.88 & 0.90 & 0.95 & 0.95 & 0.96 \\
\hline 38 & 0.94 & 0.90 & 0.92 & 0.92 & 0.96 & 0.93 & 0.94 & 106 & 0.97 & 0.95 & 0.90 & 0.88 & 0.94 & 0.93 & 0.91 \\
\hline 39 & 0.97 & 0.94 & 0.95 & 0.91 & 0.96 & 0.93 & 0.94 & 108 & 0.98 & 0.94 & 0.94 & 0.94 & 0.97 & 0.95 & 0.96 \\
\hline 40 & 0.95 & 0.95 & 0.94 & 0.94 & 0.96 & 0.93 & 0.95 & 109 & 0.97 & 0.95 & 0.91 & 0.91 & 0.95 & 0.92 & 0.94 \\
\hline 41 & 0.96 & 0.93 & 0.92 & 0.91 & 0.96 & 0.92 & 0.96 & 110 & 0.95 & 0.96 & 0.94 & 0.93 & 0.96 & 0.94 & 0.95 \\
\hline 42 & 0.96 & 0.88 & 0.88 & 0.87 & 0.94 & 0.92 & 0.96 & 111 & 0.95 & 0.94 & 0.89 & 0.93 & 0.97 & 0.95 & 0.95 \\
\hline 43 & 0.97 & 0.96 & 0.90 & 0.92 & 0.97 & 0.93 & 0.96 & 112 & 0.97 & 0.97 & 0.93 & 0.93 & 0.95 & 0.94 & 0.95 \\
\hline 44 & 0.97 & 0.95 & 0.94 & 0.92 & 0.96 & 0.93 & 0.95 & 114 & 0.97 & 0.95 & 0.93 & 0.93 & 0.97 & 0.93 & 0.97 \\
\hline 45 & 0.97 & 0.94 & 0.94 & 0.92 & 0.96 & 0.94 & 0.94 & 115 & 0.96 & 0.94 & 0.93 & 0.93 & 0.96 & 0.94 & 0.97 \\
\hline 46 & 0.97 & 0.96 & 0.96 & 0.94 & 0.97 & 0.97 & 0.98 & 116 & 0.96 & 0.96 & 0.94 & 0.94 & 0.96 & 0.94 & 0.97 \\
\hline 47 & 0.97 & 0.95 & 0.94 & 0.94 & 0.95 & 0.94 & 0.95 & 117 & 0.96 & 0.94 & 0.94 & 0.91 & 0.97 & 0.94 & 0.95 \\
\hline 50 & 0.98 & 0.94 & 0.94 & 0.94 & 0.97 & 0.95 & 0.97 & 118 & 0.94 & 0.95 & 0.94 & 0.95 & 0.96 & 0.94 & 0.94 \\
\hline 51 & 0.98 & 0.95 & 0.95 & 0.94 & 0.97 & 0.96 & 0.98 & 120 & 0.97 & 0.95 & 0.92 & 0.94 & 0.96 & 0.93 & 0.95 \\
\hline 52 & 0.97 & 0.95 & 0.94 & 0.96 & 0.98 & 0.95 & 0.97 & 121 & 0.97 & 0.96 & 0.95 & 0.95 & 0.97 & 0.95 & 0.97 \\
\hline 53 & 0.96 & 0.94 & 0.93 & 0.94 & 0.95 & 0.93 & 0.95 & 122 & 0.97 & 0.96 & 0.96 & 0.95 & 0.96 & 0.93 & 0.96 \\
\hline 54 & 0.95 & 0.94 & 0.92 & 0.89 & 0.95 & 0.93 & 0.95 & 123 & 0.97 & 0.97 & 0.95 & 0.94 & 0.96 & 0.96 & 0.97 \\
\hline 55 & 0.97 & 0.96 & 0.95 & 0.93 & 0.97 & 0.95 & 0.96 & 124 & 0.97 & 0.97 & 0.95 & 0.94 & 0.97 & 0.92 & 0.94 \\
\hline 57 & 0.98 & 0.96 & 0.95 & 0.94 & 0.97 & 0.95 & 0.95 & & & & & & & & \\
\hline 58 & 0.98 & 0.96 & 0.95 & 0.94 & 0.98 & 0.97 & 0.98 & & & & & & & & \\
\hline 59 & 0.97 & 0.96 & 0.93 & 0.92 & 0.96 & 0.94 & 0.97 & Min & 0.89 & 0.88 & 0.86 & 0.87 & 0.86 & 0.86 & 0.90 \\
\hline 60 & 0.98 & 0.97 & 0.95 & 0.94 & 0.97 & 0.94 & 0.97 & Max & 0.98 & 0.98 & 0.96 & 0.96 & 0.98 & 0.98 & 0.98 \\
\hline 61 & 0.95 & 0.92 & 0.93 & 0.94 & 0.93 & 0.91 & 0.96 & Mean & 0.96 & 0.95 & 0.93 & 0.93 & 0.95 & 0.93 & 0.95 \\
\hline
\end{tabular}

Absolute power values were compared between the first 10 2-s long EEG epochs and the last 10 2-s long EEG epochs.

\section{REFERENCES}

Eisenegger, C., Knoch, D., Ebstein, R. P., Gianotti, L. R., Sandor, P. S., and Fehr, E. (2010). Dopamine receptor D4 polymorphism predicts Mazur, J. E. (1987). "An adjusting the effect of L-DOPA on gambling behavior. Biol. Psychiatry 67, 702-706. pazur, J. E. (1987). "An adjusting
procedure for studying delayed reinforment," in Quantitative Analyses of Behavior, eds M. L.
Commons, J. E. Mazur, J. A. Nevin, and H. Rachlin (Hillsdale, NJ: Lawrence Erlbaum Associates), 55-73. 